\title{
Augmenting Forearm Crutches with Wireless Sensors for Lower Limb Rehabilitation
}

\author{
Geoff V. Merrett ${ }^{\mathrm{a},}$, , Mohamed A. Ettabib $^{\mathrm{a}}$, Christian Peters ${ }^{\mathrm{b}}$, Georgina Hallett ${ }^{\mathrm{c}}$, Neil M. White ${ }^{\mathrm{a}}$ \\ ${ }^{a}$ Pervasive Systems Centre, School of Electronics and Computer Science, University of Southampton, SO17 1BJ, UK \\ ${ }^{\mathrm{b}}$ Institut für Mikro- und Sensorsysteme, Otto-von-Guericke-Universität Magdeburg, D-39106 Magdeburg, Germany \\ ${ }^{\mathrm{c}}$ Physiotherapy Department, Southampton University Hospitals NHS Trust, Southampton, SO16 6YD, UK \\ ${ }^{*}$ Corresponding author: Tel.: +44 (0)23 8059 2775; Fax.: +44 (0)23 8059 2901; E-mail: gvm@ecs.soton.ac.uk (G.V. Merrett)
}

\begin{abstract}
Forearm crutches are frequently used in the rehabilitation of an injury to the lower limb. The recovery rate is improved if the patient correctly applies a certain fraction of their body weight (specified by a clinician) through the axis of the crutch, referred to as partial weight bearing (PWB). Incorrect weight bearing has been shown to result in an extended recovery period or even cause further damage to the limb. There is currently no minimally-invasive tool for long-term monitoring of a patient's PWB in a home environment. This paper describes the research and development of an instrumented forearm crutch that has been developed to wirelessly and autonomously monitor a patient's weight bearing over the full period of their recovery, including its potential use in a home environment. A pair of standard forearm crutches are augmented with low-cost off-the-shelf wireless sensor nodes and electronic components to provide indicative measurements of the applied weight, crutch tilt, and hand position on the grip. Data are wirelessly transmitted between crutches and to a remote computer (where they are processed and visualized in LabVIEW), and the patient receives biofeedback by means of an audible signal when they put too much or too little weight through the crutch. The initial results obtained highlight the capability of the instrumented crutch to support physiotherapists and patients in monitoring usage.
\end{abstract}

\section{Keywords}

assistive healthcare, forearm crutches, instrumented objects, biomedical engineering, patient monitoring, biofeedback

\subsection{Introduction}

Forearm crutches are used routinely following many operations to the lower limb (including the repair of fractures and the fixation of implants) in order to reduce weight-bearing through the affected limb and optimize the healing conditions for bone and soft tissues. It is widely recognized that excessive loading of the lower limb following certain types of surgery can disrupt the operated tissues and put the healing bones at risk of mal-union, while mobilisation soon after surgery increases the bone turnover metabolism and stimulates bone growth [1]. It has also been recognized that prolonged unloading of the articular cartilage causes the cartilage to become less stiff and less able to tolerate high loads [2]. Therefore, a programme of protective partial weight bearing (PWB) usually begins immediately after certain types of surgery and continues until full weight bearing is achieved at a time when there is sufficient healing in the limb. The level of PWB prescribed by the clinician (ranging from non weight bearing to full weight bearing) is dependent upon the severity and nature of the injury, the method of surgical intervention, and stage in the healing process [3]. It is critical that the patient follows this program in order to expedite the rehabilitation period and avoid further and long-term damage to the affected limb.

To ensure that the patient loads their affected limb at the prescribed level, they receive PWB training from a clinician before they are discharged from hospital [4]. As a patient's perception of the loading on their lower limb is usually prone to considerable error, a range of training techniques exist. These include the use of bathroom scales $[1,3,5-8]$, visual examination by a clinician $[4,8,9]$, subjective measurement by a clinician whose hand is placed under the patient's foot 
$[7,8]$, video instruction [10], full-length mirrors [5], force platforms [8], and in-shoe pressure monitors [4, 8]. In practice, visual examination is widely used, which introduces considerable error in the loading of the affected limb.

The effectiveness of 'static' training tools (such as bathroom scales) is disputed, as the ability to correctly perform PWB in a static posture may not necessarily transfer to dynamic gait $[7,10]$. During gait, the force through the lower limb can range between zero and five times body weight, dependent on the speed and stride length $[8,11]$. It is reported that tools that monitor the patient during gait and provide biofeedback allow more accurate, objective and reliable data [8]. However even with the use of these objective training tools, the patient's ability to remember how to correctly use the crutches is limited [10]. Therefore, the effectiveness of PWB is questionable, with patients usually and regularly overloading the affected limb once they return to a home environment [1, 4, 9]. As an example, one study found that patients were putting, on average, $36 \%$ of their body weight through their affected limb when they had been prescribed a target PWB of $10 \%$ [3]. This study also found that patients are better at maintaining a PWB of $50 \%$ of their body weight through their affected limb, as opposed to the extremes of $10 \%$ and $90 \%$. Aside from simply forgetting how to correctly perform PWB, a patient may apply too little weight when in they are in pain, or too much when pain has subsided (possibly caused by a high dose of analgesia) [6]. It has also been reported that the absence of a clinician, the home environment, the time since surgery, and the many routine distractions of daily life can all influence the ability of the patient to comply with their recommended PWB programme [4].

A number of biofeedback devices have been developed in the past that monitor the weight being exerted through the patient's shoes $[8,12]$; these are generally invasive as they require the patient to wear special footwear or attach devices to their own shoes. The concept of an instrumented walking aid has been previously investigated; Engel et al. [13] modified a walking cane to monitor PWB using a compressible spring inside the tube of the cane which activated (adjustable) micro switches when too much or too little force was applied. Biofeedback was provided to the patient by means of a vibrating cane handle, and to the clinician via two LEDS on the handle. Bergmann et al. [14] used a similar 'mechanical' sensing system to monitor the force exerted through a forearm crutch, but added a mercury tilt switch to infer the phase of the gait cycle. Neither of these systems offered telemetry or were able to perform signal analysis on the sensed data and, arguably, the presence of a spring affected the patient's usage of the crutch. Clearly, in this application domain, untethered operation is essential, and recent advances in wireless sensing and sensing networks clearly lend themselves to providing this functionality and improving the usability of devices. Wu et al. [15] developed a 'smart cane' for geriatrics to monitor the usage of the cane and infer further information about the patient's well-being. Raw data from various sensors on the cane are wirelessly streamed to a PDA via Bluetooth to provide analysis and feedback, for example detecting incorrect usage or if the patient has fallen. The requirements for an instrumented crutch are largely distinct from this as, unlike a walking cane which is used to assist full-weight-bearing gait only, the forearm crutch is used for PWB to reduce and control the loading on the lower limb following an injury. Šantić et al. [16] produced a system that monitored the forces through the crutches (using an infrared transducer) and feet (using capacitive force sensors strapped to the shoes). The system used infrared telemetry to communicate data from the sensors requiring multiple receivers to be present in the environment, hence rendering it unsuitable for use in a patient's natural environment. While earlier research has also developed instrumented crutches for analyzing kinematics and gait within a laboratory environment [17], our proposed device is designed for biofeedback in assistive healthcare (i.e. to be used for the patient's benefit throughout the entirety of their rehabilitation programme).

In this paper, we present the research, design, and development of a pair of forearm crutches augmented with low-cost wireless sensors for use in both in-hospital training and patient monitoring over the full period of recovery (including its potential use in the home environment). The crutches monitor the force being applied through their axis, enabling an indicative and objective estimation of the weight being exerted through the affected limb to be obtained. To assist in teaching patients the correct usage of forearm crutches, the tilt of the crutch and position of the hand on the grip are also measured. Two crutches are required for PWB, and both must be instrumented in order to account for patients which may unevenly distribute their weight through the crutches (although not ideal, this is quite likely to occur due to pain and an instinctive protection of the affected leg). The developed crutch was designed as a research tool for physiotherapists at Southampton General Hospital, and the specification and design of the crutch was undertaken with considerable input from them. The physiotherapist can observe data from the crutch in real-time using a LabVIEW graphical user interface (GUI) on a remote computer, while the patient receives biofeedback by means of an audible notification when PWB events occur (i.e. too much or too little weight is exerted through the affected limb). The instrumented crutch provides 
clinicians and patients with a means of objectively measuring and receiving feedback on the weight being exerted through their affected limb. Such an instrumented crutch differs from those previously researched and developed for PWB by being less-invasive (no additional equipment is required to be attached to the patient or their footwear, as all electronics is contained within the crutch), and being used for both clinical training and long-term in-home monitoring.

\subsection{Concept and System Architecture}

Through consultation with clinicians at Southampton General Hospital, it was specified that the primary aim of an instrumented crutch would be to assist in both training and long-term monitoring of a patient's PWB programme. The secondary aim was to infer information about how the patient is using the crutch. The system should augment a standard low-cost pair of forearm crutches, thus dictating the use of off-the-shelf components. The low-cost requirement also typically infers a low level of accuracy. However, in this application the required level of accuracy was identified to be $<5 \%$, a level which would provide a significant patient benefit over existing methods and systems. Any improvement in this accuracy is likely to be unnecessary, as alternative measurement errors are likely to become predominant as a result of the force distribution through the bones and soft tissues. It was also stressed however that the system needed to be easy to use and simple to configure in order for it to achieve acceptance by both patients and clinicians alike. Figure 1 shows the uses of the crutch, including real-time observation of data by the clinician (to train patients how to use the crutch) and to provide real-time biofeedback to the patient (encouraging them to consistently put the recommended weight through the limb). Clearly a wireless, low-power, small and lightweight system is essential for such an application.

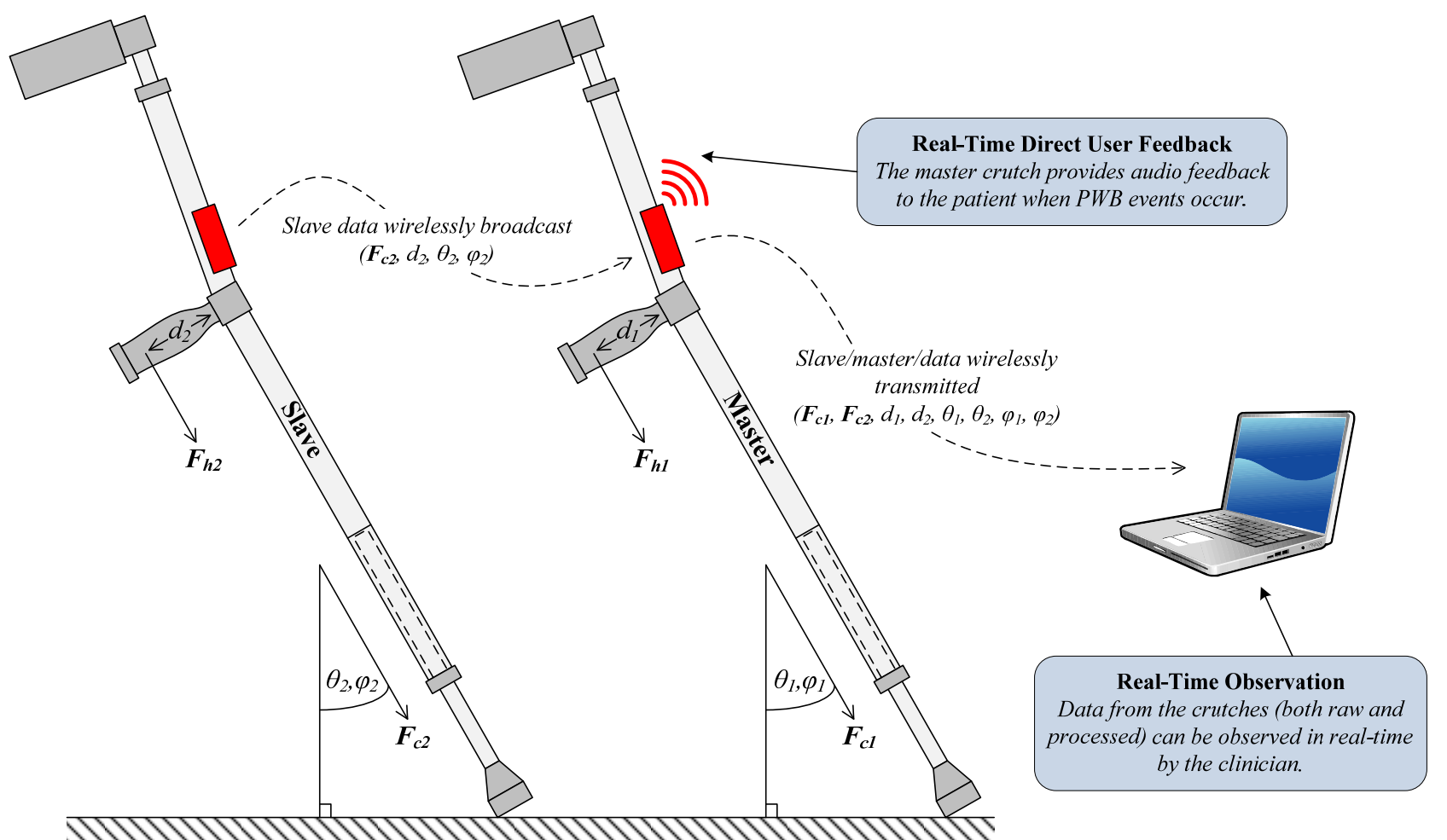

FIGURE 1: The instrumented crutch, showing the architecture (crutch-to-crutch, or crutch-to-host), functionality (realtime observation and biofeedback), and also the forces, angles, and distances measured.

To achieve the primary aims (monitoring PWB), it was realised that the crutch should monitor the magnitude of the force translated through the axis of the crutch $\left(\left|F_{c}\right|\right.$ in Figure 1) thus allowing the weight-bearing of the affected limb to be estimated. The secondary aims of the system require the measurement of the crutch tilt (the angles between the crutch and the ground parallel and perpendicular to the walking direction, i.e. the pitch, $\theta$, and roll, $\varphi$, components of the unit vector $\left.\widehat{F}_{c}\right)$, and an indicative measurement of the patient's grip pattern - implemented through identifying the position $(d)$ at which the grip force $\left(F_{h}\right)$ is applied to the handle. 


\subsection{The Instrumented Crutch}

This section describes the various hardware and software components of the instrumented crutches. One crutch acts as a master; the master crutch receives data from the other crutch, referred to as the slave, and processes the data to provide biofeedback to the patient via an audible buzzer.

\subsection{Hardware Architecture}

The hardware consists of low cost sensors integrated into the crutch for measuring $\left|F_{c}\right|, \theta, \varphi$ and $d$, and a low power embedded microcontroller and radio transceiver to sample, process and communicate data between the crutches and back to the host computer. The crutches were developed using commercial off-the-shelf (COTS) components in order to minimise the potential cost. As is visible in Figure 2, the crutch hardware is currently in prototype; it is envisaged that all hardware (batteries, cabling, sensors and circuitry) can all be housed within the crutch pole, with the possible exception of the radio antenna.

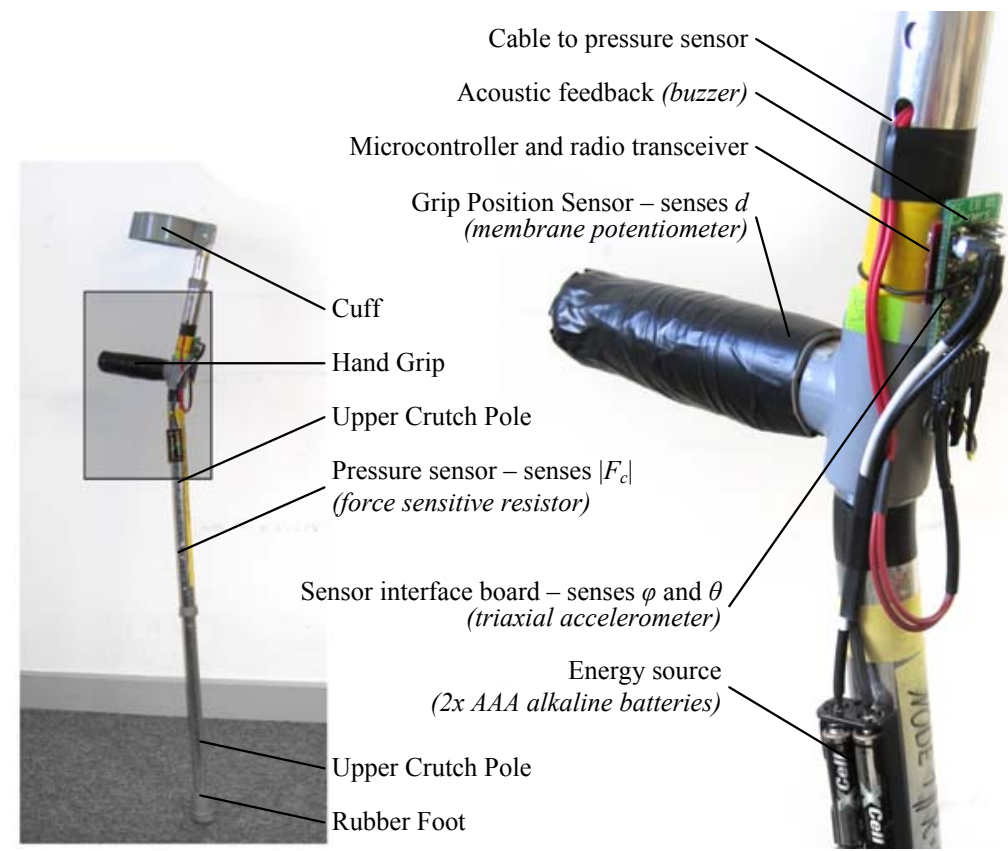

FIGURE 2: One of the instrumented crutches, showing the locations of the major components and parts.

The centre of the instrumented crutch is a Texas Instruments ez430-rf2500 wireless development tool [18]. This device contains both a 16-bit MSP430 low-power microcontroller and a CC2500 2.4GHz ultra-low-power radio transceiver. The host computer (which can receive data from both crutches and display it to a clinician in real-time) uses an identical ez430-rf2500 wireless development tool, connected via a USB port. The CC2500 radio transceiver communicates in the $2.4 \mathrm{GHz}$ International Industrial, Scientific and Medical (ISM) band, with a radiated output power of $0 \mathrm{dBm}$. Communication is performed using the proprietary SimpliciTI [19] low-power network protocol from Texas Instruments. Raw data from the sensors are broadcast from the slave crutch and received by the master crutch and, when enabled, both master and slave crutches independently establish a connection with the host computer to communicate data. Due to a requirement for fast sampling, all data are communicated without acknowledgments.

To enable the crutches to be used out of the hospital and in a home environment, it is essential that they can operate for prolonged periods of time through use of their onboard batteries. Hence, energy efficient operation of the crutches is of significant importance. A commonly used method of reducing the average current consumption is to duty cycle the microcontroller and/or radio transceiver into low-power sleep states. As the crutch is continuously sampling and processing data from its sensors, it is not possible to put the microcontroller into a sleep state (unless the sampling rate was reduced to permit this). Further, using the existing communication scheme it is also not possible to duty cycle the radio transceiver of the master crutch, as this continuously listens for packets transmitted by the slave crutch. The crutches can be turned off when not in use by detaching the power connector or removing the batteries. To further reduce 
the power consumption, communication to the host computer is not enabled when the crutches are turned on. To enable this mode of communication, a micro-switch on each crutch must be depressed while they are powered on. The radio transceiver on the slave node is duty cycled, and enters a sleep state whenever it is not communicating with the master crutch. The crutches are powered from two 1175mAh AAA-sized batteries mounted on the crutch shaft, with the aim of sustaining operation for at least 24 hours.

A dedicated sensor interface PCB is connected to the rear of the ez430-rf2500 via general purpose IO lines, and contains interface circuitry for sampling the various sensors on the crutch. Data from the sensors are sampled via the microcontroller's onboard 10-bit analogue-to-digital convertor (ADC) and operational amplifiers. A continuous loop operates in the microcontroller's embedded software, in which data are sampled from each sensor (the FSR, potentiometer, and three individual axis of the accelerometer), processed accordingly and, in the case of the slave crutch, communicated to the master. Additionally, if enabled, data are also communicated to the host computer). This sequence of operations repeats at a frequency of $38 \mathrm{~Hz}$.

The primary purpose of the instrumented crutch is to measure the force applied through its axis, i.e. the ground reaction force (GRF) or $F_{c}$ in Figure 1. In order to obtain a measurement of the GRF, both Force Sensitive Resistors (FSRs) and strain gauges were considered. An FSR was selected as the most suitable method due to their low-cost and ease of retrofitting (strain gauges would need to measure compression and tension forces in the sides of the crutch pole as opposed to the direct compression through the crutch axis; this would require an complex adhesion procedure and protective housing in order to practically assemble it). The magnitude of the GRF, $\left|F_{c}\right|$, is measured using a FlexiForce ${ }^{\circledR}$ FSR [20] mounted inside the crutch pole (the location of which is shown in Figure 3). This location was selected, as opposed to mounting the FSR at the bottom of the crutch on the inside of the rubber foot, to minimise possible adverse issues caused by friction or compression of the foot. The FSR was conditioned assuming a maximum force of $1 \mathrm{kN}$ (equivalent to a patient's weight of $\approx 100 \mathrm{~kg}$ ) and the force-conductance relationship linearly characterized over this range with a relative error of $\leq 10 \%$. Naturally, issues including repeatability and drift are to be experienced, but are manageable through regular zeroing and self-calibration (discussed later) and the relaxed accuracy requirement inferred by the application.

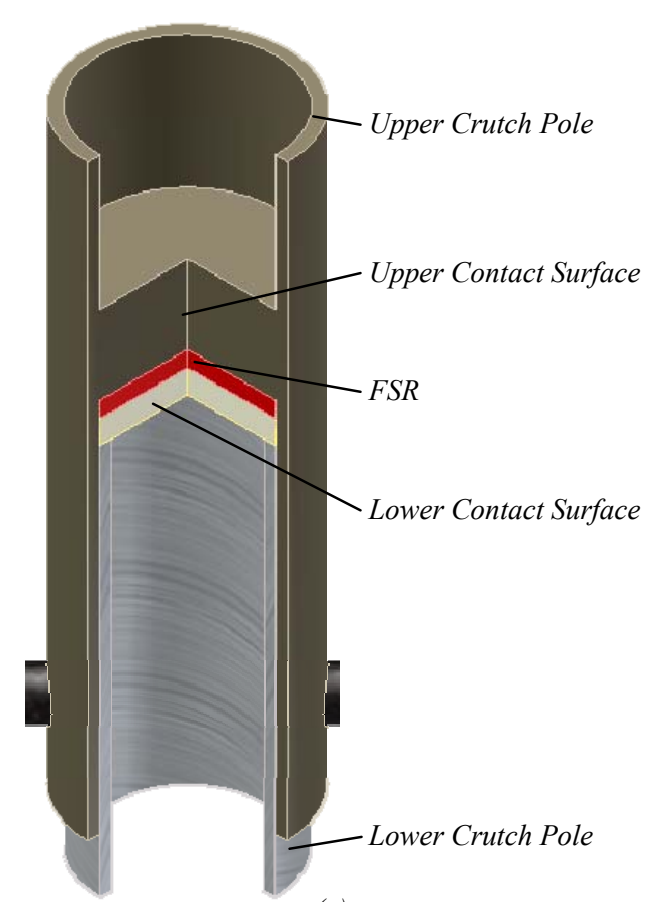

(a)

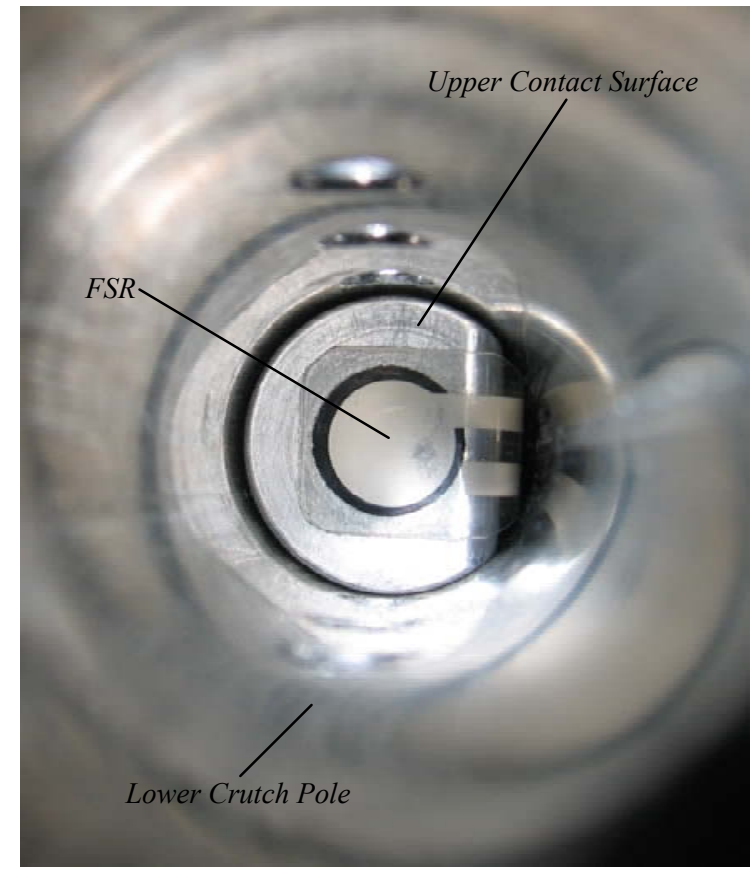

(b)

FIGURE 3: a) A drawing showing the location of the FSR (measuring $F_{c}$ ) inside the main crutch pole, and b) a photograph of the FSR located inside the crutch (taken looking up the main crutch pole before the lower crutch pole is inserted).

The crutch tilt, $\widehat{F}_{c}$, is measured using an STmicro LIS3LV02DL MEMS tri-axial accelerometer [21] and calculated with the assumption that 'passive' accelerations (accelerations due to gravity) are predominant over 'dynamic' accelerations 
(accelerations due to a rapid change in velocity, such as shocks, movement and vibrations). In reality, the presence of dynamic accelerations gives rise to occasional acceptable errors, and can be minimised through the inclusion of a lowpass filter. The magnitude of dynamic accelerations is also likely to increase with a faster walking speed, and hence increases the error in calculating $\widehat{F}_{c}$. However, in a clinical environment patients are taught to use crutches at a safe speed, which is anecdotally slower than normal walking speed and usually quite deliberate and careful. As the crutch therefore assumes that only passive accelerations, the accelerometer is configured in the low- ' $\mathrm{g}$ ' mode $\left( \pm 2^{\prime} \mathrm{g} g\right.$ '). In order to calibrate the accelerometer (required for calculating the tilt angles) the system's GUI can be used to enter values for each component at $-1,0$ and 1 ' $\mathrm{g}$ ' (obtained by rotating the crutch to specific orientations).

Finally, the distance at which the grip force is applied to the handle, $d$, is measured using a SpectraSymbol $10 \mathrm{k} \Omega$, $100 \mathrm{~mm}$ rectilinear membrane potentiometer [22] mounted inside the hand grip. The sensor is located underneath the rubberised cover to protect it from adverse impacts and damage, and the plastic inner-bar was coated in epoxy composite and smoothed to remove any unevenness in the mounting surface. The signal is converted into a voltage using an inverting amplifier, from which an estimate of the position of the hand on the grip can be obtained. Using a foil potentiometer, the value obtained for $d$ provides an indication of incorrect grip position, through the identification of the force applied closest to the crutch axis. The distance, $d$, and the crutch tilt, $\widehat{F}_{c}$, are not used to estimate the weight applied through the affected limb, but provide supplementary information on crutch usage (requested by clinicians during our initial consultations).

\subsection{Software Architecture}

The software system (shown in Figure 4) of the instrumented crutches consists of the embedded software on the master and slave crutches, the embedded software on the ez430-rf2500 connected to the host computer via USB (this is not discussed further as its operation is only to receive data from the crutches and pass it into a virtual serial port on the host computer), and the LABView graphical user interface for recording and visualising data from the crutches in real-time. The embedded software on the ez430-rf2500s is programmed in C using the Texas Instruments Code Composer Essentials integrated development environment.

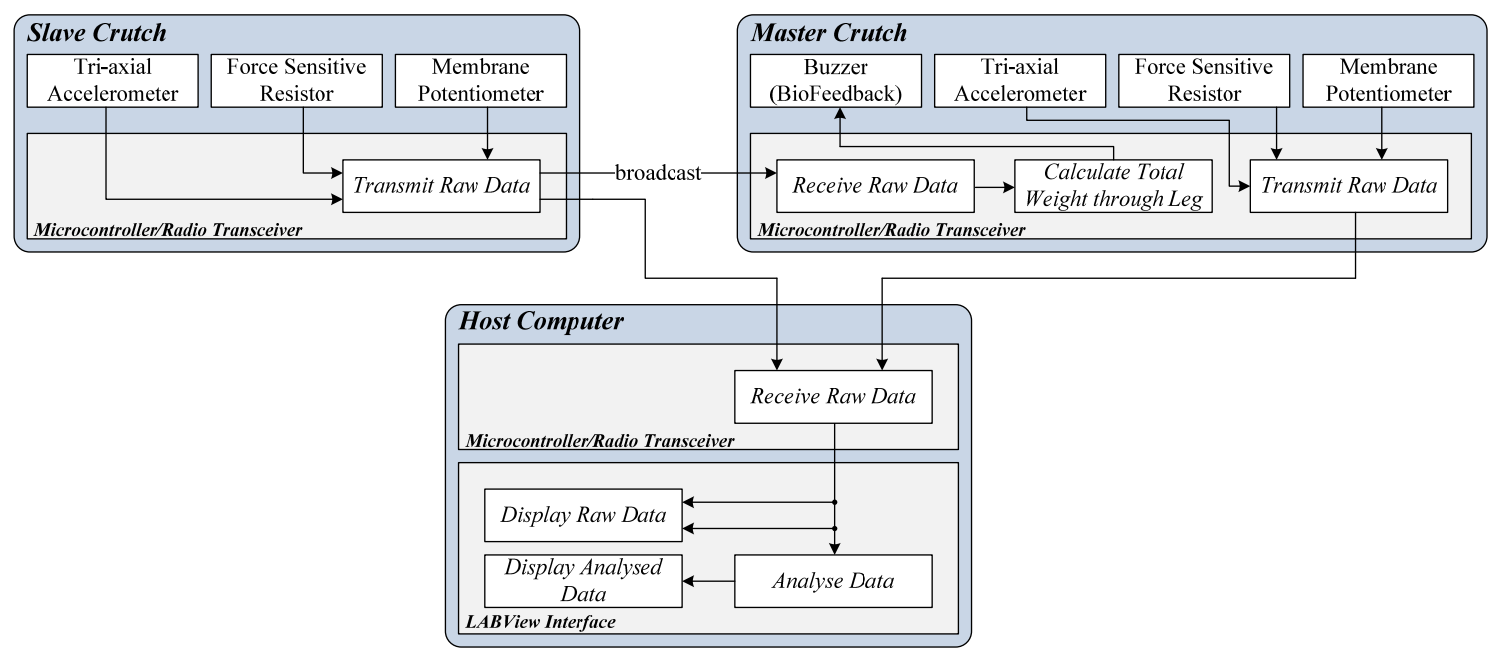

FIGURE 4: Data flow through the instrumented crutch system.

The slave crutch samples its various sensors and transmits these raw data, unprocessed from the output of the ADC, to the master crutch. The master crutch receives these data and, by fusing it with samples taken from its own sensors, calculates the level of PWB (providing biofeedback when necessary). If enabled, both crutches also independently transmit their raw sensor data to the host computer.

\subsubsection{Embedded Processing}

Having sampled the data from its own sensors and receiving data from the slave crutch's sensors, the master crutch estimates the weight through the affected limb in order to provide biofeedback. To understand the reasoning behind this algorithm, consider a typical PWB gait cycle as depicted by Figure 5. If the maximum force through the patient's 
affected limb is identified, this considers the impact forces (heal contact and propulsion) as being relevant to the process of PWB. While this could be seen to be of importance (as these forces are being translated through the affected limb), the decision was taken in this research that it was not the force that was of interest. This is because PWB is prescribed as a fraction of the patient's body weight, and hence is normalised to $100 \%$ as 'normal' gait. Hence, impact forces are part of a patient's normal gait pattern, and the instrumented crutch needs to identify where the prescribed PWB limit applies. For this reason, the algorithm identifies and uses the force in the midstance phase (shown in Figure 5), as it is at this point that the full extent of the patient's weight is applied through the crutches and the affected limb (i.e. the affected limb is in the midstance phase, and the healthy limb is most likely to be off the ground and not under load). It is also at this point that the limb is under a 'steady' force (and not a dynamic heal contact or propulsion force). These decisions were made upon consultation with clinicians in order to identify which forces were of specific interest. The midstance force occurs when the force through the crutches are at their maximum.

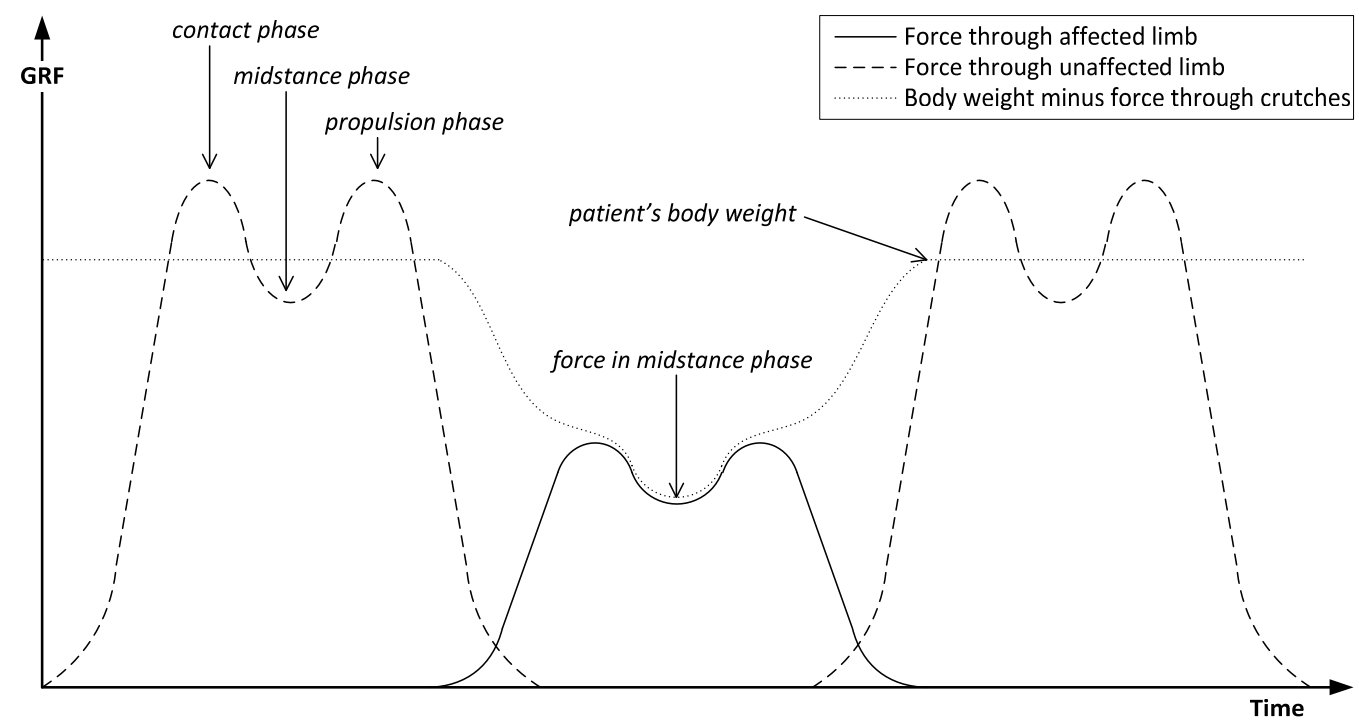

FIGURE 5: Graphical depiction of the theoretical forces through the affected limb, healthy limb, and crutches during PWB crutch-assisted gait.

As discussed in the introduction, it is recognised that the maximum GRF is highly correlated with the walking speed and stride length, for example where impact forces increase with an increase in walking speed. By neglecting the impact forces in our calculations, this correlation is likely to be less significant as the 'steady' force will still relate to the fraction of body weight being applied through the affected limb. Further, a patient's walking speed should also be reasonably limited as the clinician will teach them to use the crutches in a slow and controlled manner.

Equation (1) is used to calculate the percentage of the patient's body weight that is translated through their limbs, where $\left|F_{c 1}[n]\right|$ and $\left|F_{c 2}[n]\right|$ are sets of the sampled magnitudes of the forces through the axes of crutches 1 and 2 during the period of one gait cycle, $M$ is the mass of the patient $(\mathrm{kg})$ and $g$ is the acceleration due to gravity $\left(\mathrm{ms}^{-2}\right)$. The maximisation is performed over the sum of the crutch forces with respect to $n$, where $n$ is a discrete time index. Note that this equation estimates only the weight translated through the patient's limbs; hence it can only provide the weight bearing through the affected limb when the healthy limb is not in contact with the ground. The use of forearm crutches in PWB gait entails planting both crutches on the ground at the same time (which is while the affected limb is in contact with the ground). Therefore, and because the algorithm uses only the total force through both crutches, there is no requirement for the master (or slave crutch) to be used on a particular side of the body. Hence the system still operates correctly if the crutches are accidently interchanged.

$$
W_{\text {actual }}^{\%}=1-\frac{\max \left(\left|\boldsymbol{F}_{c 1}[n]\right|+\left|\boldsymbol{F}_{c 2}[n]\right|\right)}{M \cdot g}
$$

The parameters entered into the smart crutch are the patient's weight $M[\mathrm{~kg}]$, the target level of PWB $W_{\%}^{\text {aim }}[\%]$, and the tolerance that is permissible in the PWB applied $\alpha[ \pm \%]$. These parameters are different for each patient and also depend on the stage that they are at in their rehabilitation programme. The crutch checks to see if biofeedback is required using 
equation (2). If the lower threshold is not met, the onboard buzzer sounds once to provide biofeedback to the patient. If the upper threshold is exceeded, the onboard buzzer sounds twice.

$$
W_{\text {target }}^{\%}-\alpha<W_{\text {actual }}^{\%}<W_{\text {target }}^{\%}+\alpha
$$

This algorithm is continually repeated at the master crutch for each gait cycle.

\subsubsection{Graphical User Interface}

The host computer is used to display the real-time data received from the crutches using a LabVIEW GUI, which allows the raw data from all sensors on both crutches to be viewed in graph form. Screenshots from the raw data and visualisation screens can are shown in Figure 6.
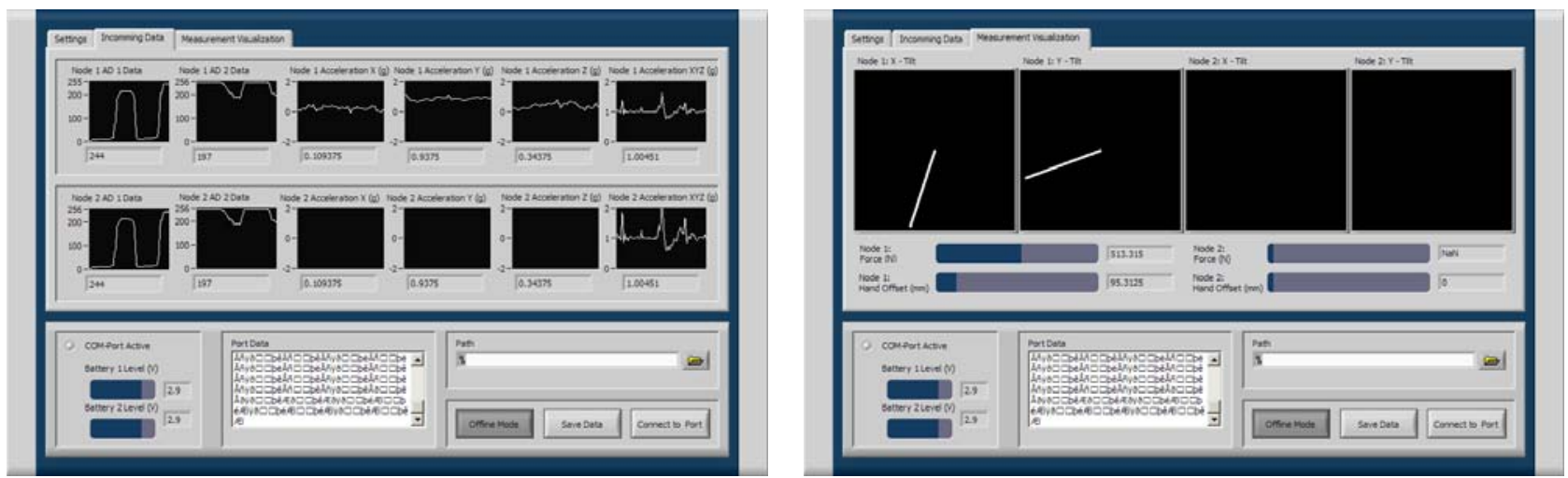

FIGURE 6: Screenshot of the LABView interface, i) the raw data screen, and ii) the visualisation screen.

The GUI also converts the ADC output values representing $\left|F_{c}\right|$ into a force [N], $d$ into a distance [mm], and $\widehat{F}_{c}$ into the total acceleration ['g'] and tilt angles $\theta$ and $\varphi$ [degrees]. Additionally, the energy remaining in the batteries in each crutch can be inspected, the data received from the crutches exported to a text file (for further analysis) or imported into the GUI (for offline viewing).

\subsection{Results and Discussion}

Figure 7 shows the data obtained from the one of the two crutches while a subject was walking with it (exported via the LabVIEW GUI), including $\left|F_{c}\right|, d, \theta$ and $\varphi$. The data obtained from angles $\theta$ and $\varphi$ have been filtered by a five-point moving average filter to attenuate dynamic accelerations. From Figure 7, the characteristic three-point gait can be identified (positive values of $\theta$ occur when the crutch is behind the subject). First (marked as phase ' $a$ '), the crutch is swung in front of the subject while their body weight is supported through their healthy limb. Then, when $\theta$ is at its minimum (marked by the line separating phase ' $a$ ' and ' $b$ '), the subject loads the crutch using their body weight $\left|F_{c}\right|$. The affected limb then gently supports a partial fraction of their body weight, while the healthy limb moves forwards (marked as phase ' $b$ '). When $\theta$ is at its maximum (marked by the line separating phase ' $b$ ' and 'c'), the subject's weight is removed from the crutch, which is subsequently swung back in front of them while their body weight is supported by the healthy limb (marked by phase 'c'). This cycle then repeats as the subject continues to walk. The visible variation in $\varphi$ is due to small changes in the roll of the crutch, and also dynamic accelerations (such as impact forces etc). The hand position distance $d$ remains reasonably constant over the 20 second period for which data is shown, as expected.

A small preliminary study was performed to evaluate the system and to assess the potential for using instrumented crutches to estimate the weight-bearing through a limb. The study recorded the magnitude of the force translated through the axis of both instrumented crutches and, using a Pedar in-shoe dynamic pressure measuring system [23], the GRF put through each foot. The Pedar system consists of a pair of thin, flat and flexible insoles (placed inside the subject's shoes) containing capacitive pressure sensors that measure the body weight being applied through each foot. The insoles are wired to a processing box strapped to the subject's waist, which is connected to a laptop computer. The healthy subjects were given the instrumented crutches to use, and instructed to walk using three-point gait for ten steps, aiming to put $50 \%$ of their body weight through their affected limb. 


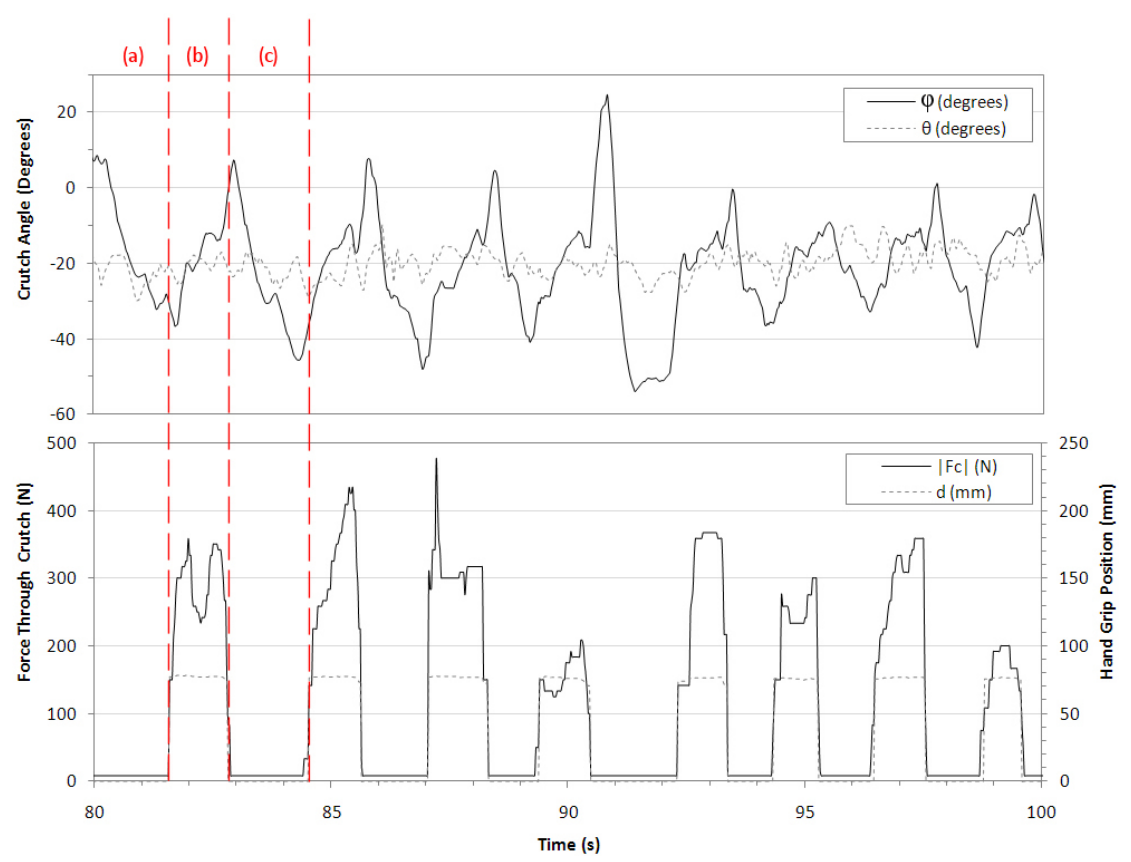

FIGURE 7: Data obtained from an instrumented crutch.

Figure 8 shows data obtained from the preliminary study, where both the affected and healthy limb's GRF is shown while attempting to PWB at 50\%. Further, the crutch's estimation of the force through the affected limb is also shown, and clearly follows changes in force through the limb correctly. As previously mentioned, because the crutches only estimate the force through any limb, the estimated force does not return to zero in-between loading the affected limb (when the crutches are not in contact with the ground). In Figure 8 there is a noticeable offset between the actual and estimated data, which has been since identified as being due to calibration issues with the FSRs. Due to the drift exhibited by the FSR (a logarithmic drift during constant loading, with a maximum of $26500 \mathrm{ppm}$ change in resistance per 1000 hours) and the sensor mountings, it will be necessary to routinely re-calibrate the crutch. From the testing that we have performed, we believe that it is reasonable to assume that the FSR has a linear force-conductance relationship (the datasheet specifies a linearity of $\pm 3 \%$ ) which passes through zero ( $0 \mathrm{~S}$ at $0 \mathrm{~N}$ ). This could be addressed by updating the embedded software on the crutches to allow them to be calibrated to within the specified tolerance in-situ by both zeroing and loading them with $500 \mathrm{~N}$ (which can be performed using body weight if no other means are available, while the crutch is on a set of scales) and pressing the micro-switch on the crutch. This can be done routinely, and recalculates the gradient of the FSR's force-conductance relationship.

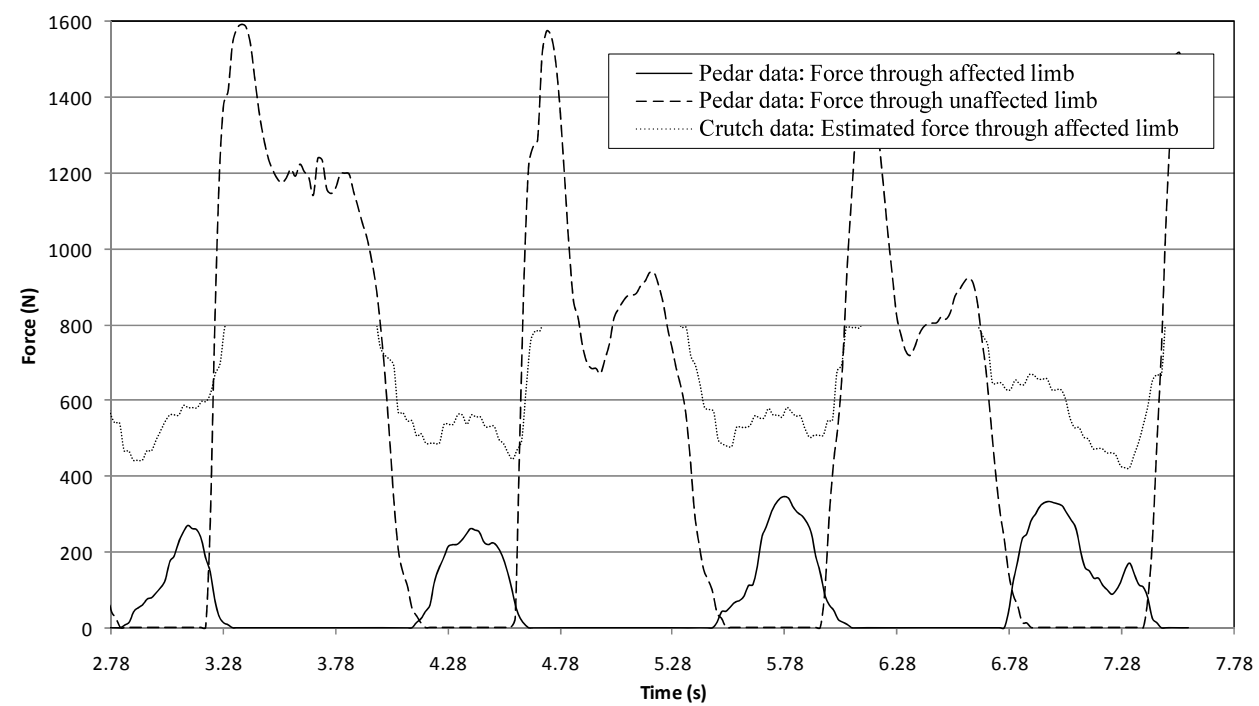

FIGURE 8: Data obtained from the preliminary study showing the GRF through both the affected and healthy limbs, and the calculated force being put through the affected limb (calculated by the instrumented crutches). 
Figure 9 shows the current consumption profile of the slave crutch, obtained using an oscilloscope measuring the voltage drop across a $10 \mathrm{Ohm}$ resistor in series with the $3 \mathrm{~V}$ power supply. As a result of successful duty cycling, the transceiver on the slave crutch is active for only $18 \%$ of the time (rising to $31 \%$ when the crutches are also communicating with the host computer). In Figure 9a, the crutch is communicating with the master and the host computer (this can be seen by the two successive communication sequences when the radio is enabled) and an LED on the crutch toggles each time the sensors are sampled. In this mode, the crutch consumes an average current of $10.2 \mathrm{~mA}$. As annotated in Figure 9a, the LED consumes $3.14 \mathrm{~mA}$ when turned on. By disabling the LED the average current consumption in this mode drops to $9.18 \mathrm{~mA}$. In a home environment, where prolonged operation is essential, communication to a host computer is not required, and in this mode the slave crutch consumes an average current of only $6.30 \mathrm{~mA}$ (shown in Figure 9b). The master crutch consumes an average current of $22.1 \mathrm{~mA}$ during operation, and the buzzer (used for biofeedback) consumes approximately $3.3 \mathrm{~mA}$ when sounding; as the patient's aim is to use the crutches without making the buzzer sound, this is omitted from our energy calculations.

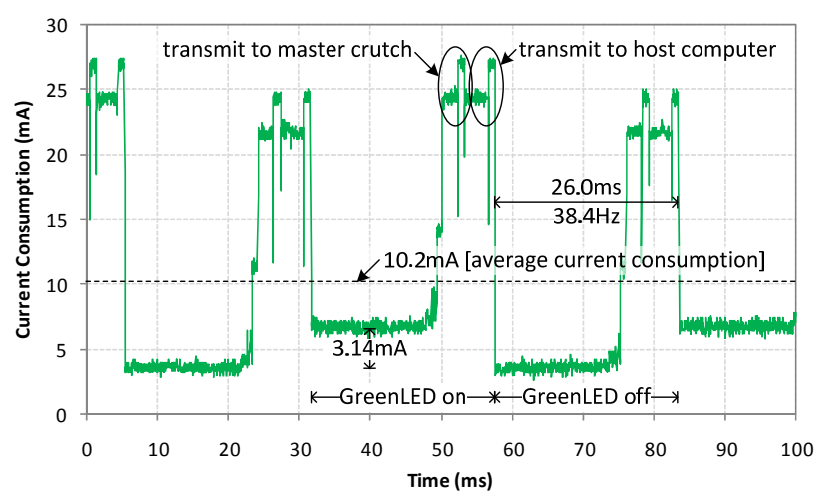

(a)

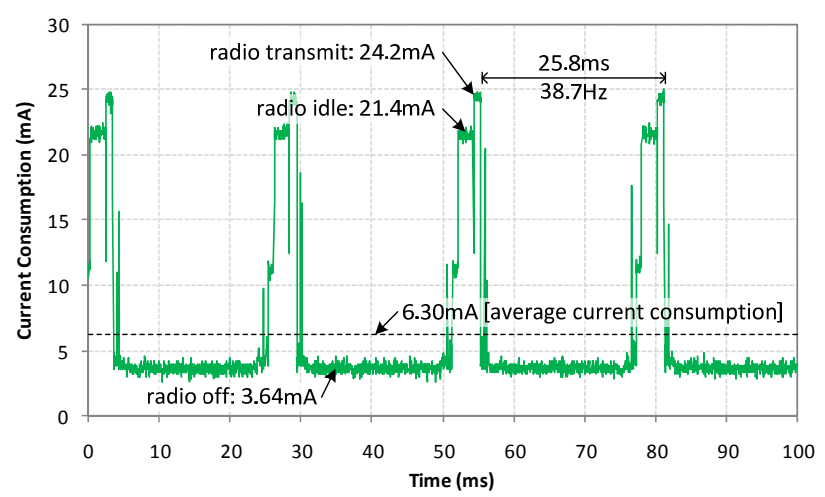

(b)

FIGURE 9: Oscilloscope traces showing the current consumption of the slave crutch when communicating with a) both the master crutch and the host computer and the LED toggles with every sample, and b) the master crutch only.

From its two 1175mAh AAA-sized batteries (providing, for low discharge currents, a total energy of around 12.7kJ), the master crutch can operate for a period of around two days, and the slave for greater than a week. Clearly, a life of greater than 24 hours is acceptable in most situations where the crutches could be recharged overnight. If six $3.6 \mathrm{~V} 2450 \mathrm{mAh}$ AA-sized batteries were used instead (these could be housed inside the lower-crutch pole), the master can operate for nearly a month, and the slave for almost 100 days between recharges. This, however, would add significant additional weight to the crutches, and hence provide a detrimental alteration to crutch usage and patient recovery. During testing, we routinely experienced successful communication between both crutches and between the crutches and the host computer over separation distances of more than $10 \mathrm{~m}$ in a cluttered indoor environment. Intuitively, this more than satisfies the application requirements.

\subsection{Conclusions and Future Work}

This paper has presented a pair of instrumented crutches to assist in partial weight bearing in orthopaedic rehabilitation. The designed crutches have clear benefits over alternative systems, including wireless data transfer, biofeedback, quantitative data and ease of configuration and setup. The preliminary results that have been obtained show that the weight-bearing of the patient can be estimated (incidentally the results also highlighted subjects' inability to repeatedly comply with PWB, agreeing with the clinical literature and the findings of our previous research [24]). However, the obtained results have also shown the need for better calibration and accuracy from the FSR, and a requirement for a more insightful understanding and prescription of the forces of interest.

Further research will also investigate the use of the accelerometer and hand grip position sensor to locally evaluate how the patient is using the crutch, and provide biofeedback on usage events (signifying incorrect usage). Furthermore, we will also investigate the use of the crutch tilt to supplement the PWB algorithm by determining when the crutches are in use and which phase of the gait cycle the affected limb is in. A data storage system on the master crutch will locally record statistics on PWB events, usage events, and the activity of the crutch (the number and speed of steps, and their distribution during the day) allowing an 'activity digest' to be downloaded and inspected by the clinician. The energy 
efficiency of the system will be improved by investigating the use of Low Power Listening [25] techniques to allow the master node to enter low-power states and balance the power consumption of master and slave nodes. Finally, a more comprehensive clinical study will be performed to fully evaluate the system.

A video showing the crutch and its use can be viewed at http://www.ecs.soton.ac.uk/podcasts/video.php?id=182.

\section{Acknowledgments}

The authors with to thank the Engineering and Physical Research Council (EPSRC) for their financial support under grant number EP/D042917/1 PLATFORM: 'New Directions for Intelligent Sensors', and Dr Cheryl Metcalf and Martin Warner (School of Health Sciences, University of Southampton) for their assistance in the preliminary study. The authors would also like to thank the anonymous reviewers for their constructive comments and ideas for future work.

\section{References}

[1] A. Vasarhelyi, T. Baumert, C. Fritsch, W. Hopfenmuller, G. Gradl, and T. Mittlmeier, "Partial weight bearing after surgery for fractures of the lower extremity - is it achievable?," Gait Posture, vol. 23, pp. 99-105, Jan 2006.

[2] J. M. Walker, "Pathomechanics and classification of cartilage lesions, facilitation of repair," J Orthop Sports Phys Ther, vol. 28, pp. 216-31, Oct 1998.

[3] S. Li, C. W. Armstrong, and D. Cipriani, "Three-point gait crutch walking: variability in ground reaction force during weight bearing," Arch Phys Med Rehabil, vol. 82, pp. 86-92, Jan 2001.

[4] H. L. Hurkmans, J. B. Bussmann, R. W. Selles, E. Benda, H. J. Stam, and J. A. Verhaar, "The difference between actual and prescribed weight bearing of total hip patients with a trochanteric osteotomy: long-term vertical force measurements inside and outside the hospital," Arch Phys Med Rehabil, vol. 88, pp. 200-6, Feb 2007.

[5] G. Wannstedt and R. L. Craik, "Clinical evaluation of a sensory feedback device: the limb load monitor," Bull Prosthet Res, pp. 8-49, Spring 1978 .

[6] A. Malviya, J. Richards, R. K. Jones, A. Udwadia, and J. Doyle, "Reproducibilty of partial weight bearing," Injury, vol. 36, pp. 556-9, Apr 2005.

[7] J. W. Youdas, B. J. Kotajarvi, D. J. Padgett, and K. R. Kaufman, "Partial weight-bearing gait using conventional assistive devices," Arch Phys Med Rehabil, vol. 86, pp. 394-8, Mar 2005.

[8] H. L. Hurkmans, J. B. Bussmann, E. Benda, J. A. Verhaar, and H. J. Stam, "Techniques for measuring weight bearing during standing and walking," Clin Biomech, vol. 18, pp. 576-89, Aug 2003.

[9] M. Tveit and J. Karrholm, "Low effectiveness of prescribed partial weight bearing. Continuous recording of vertical loads using a new pressure-sensitive insole," J Rehabil Med, vol. 33, pp. 42-6, Jan 2001.

[10] D. Krause, M. Wunnemann, A. Erlmann, T. Holzchen, M. Mull, N. Olivier, and T. Jollenbeck, "Biodynamic feedback training to assure learning partial load bearing on forearm crutches," Arch Phys Med Rehabil, vol. 88, pp. 901-6, Jul 2007.

[11] F. Polak, "Gait Analysis," in Rehabilitation of Movement: Theoretical Basis of Clinical Practice, J. Pitt-Brooke, Ed.: Bailliere Tindall, 1997, pp. 285-316.

[12] S. L. Wolf and J. E. Hudson, "Feedback Signal Based Upon Force and Time-Delay - Modification of the Krusen Limb Load Monitor," Physical Therapy, vol. 60, pp. 1289-1290, 1980.

[13] J. Engel, A. Amir, E. Messer, and I. Caspi, "Walking cane designed to assist partial weight bearing," Arch Phys Med Rehabil, vol. 64, pp. 386-8, Aug 1983 .

[14] G. Bergmann, R. Kolbel, A. Rohlmann, and N. Rauschenbach, "Walking with walking aids. III. Control and training of partial weightbearing by means of instrumented crutches," Z Orthop Ihre Grenzgeb, vol. 117, pp. 293-300, Jun 1979.

[15] W. Wu, L. Au, B. Jordan, T. Stathopoulos, M. Batalin, W. Kaiser, A. Vahdatpour, M. Sarrafzadeh, M. Fang, and J. Chodosh, "The SmartCane system: an assistive device for geriatrics," in Proceedings of the ICST 3rd international conference on Body area networks, Tempe, Arizona, 2008.

[16] A. Santic, V. Bilas, and I. Lackovic, "A system for measuring forces in the legs and crutches from ambulatory patients," in Proc. 19th Annual Int'l Conf. IEEE Engineering in Medicine and Biology Society, 1997, pp. 1895-1898.

[17] A. B. Liggins, D. Coiro, G. W. Lange, T. E. Johnston, B. T. Smith, and J. J. McCarthy, "The case for using instrumented crutches during gait analysis," in Bioengineering Conference, 2002. Proceedings of the IEEE 28th Annual Northeast, 2002, pp. 15-16.

[18] T. Instruments, "ez430-rf2500 Development Tool User's Guide," Texas Instruments SLAU227E, April 20092009.

[19] Texas Instruments, "SimpliciTI Compliant Protocol Stack." [Online]. Available: http://focus.ti.com/docs/toolsw/folders/print/simpliciti.html. [Accessed: Oct, 2009].

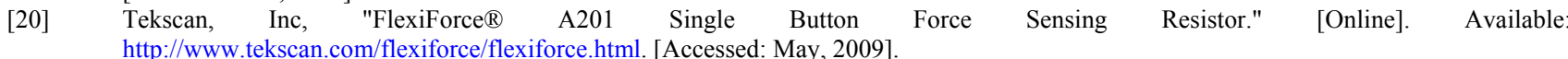
"LIS3LV02DL MEMS 3-axis $\pm 2 \mathrm{~g} / \pm 6 \mathrm{~g}$ digital output low voltage linear accelerometer," January. [Online]. Available: http://www.st.com/stonline/products/literature/ds/12094.pdf. [Accessed: September, 2009].

[22] "Spectra Symbol SoftPot Foil Potentiometers." [Online]. Available: http://www.spectrasymbol.com/typo3/site/en/softpotsplash/softpot.html. [Accessed: September, 2009].

[23] Novel GmbH, "Pedar Systems." [Online]. Available: http://www.novel.de/productinfo/systems-pedar.htm. [Accessed: Oct, 2009].

[24] G. V. Merrett, C. Peters, G. Hallett, and N. M. White, "An Instrumented Crutch for Monitoring Patients' Weight Distribution during Orthopaedic Rehabilitation," in Eurosensors XXIII Lausanne, Switzerland: Elsevier Procedia Chemistry, 2009.

[25] J. Polastre, J. Hill, and D. Culler, "Versatile low power media access for wireless sensor networks," in Int'l Conf. Embedded Networked Sensor Systems (SenSys'04), Baltimore, MD, United States, 2004, pp. 95-107. 